PHYSICAL CHEMISTRY

\section{Seeds of phase change}

\author{
Daan Frenkel
}

\section{An effective but counter-intuitive trick to obtain highly ordered protein crystals is to 'seed' particles on disordered, porous surfaces. Computer simulations provide an explanation for the success of this strategy.}

The highly ordered, three-dimensional arrays of molecules that we know as crystals can form spontaneously from the disordered liquid phase. To speed up the rate of crystal formation, crystal-growers often use 'seeds' - templates that can kick-start crystallization. A few years ago, experiments on protein crystallization $^{1}$ unexpectedly showed that highly disordered, porous particles can be particularly effective in initiating the growth of perfectly ordered crystals. Writing in Physical Review Letters $^{2}$, Page and Sear now explain why the disorder of porous templates allows them to behave as universal crystallization agents.

In many areas of science and technology, it is essential to control crystallization. For example, proteins must first be crystallized if we are to determine their structures from X-ray crystallography; pharmaceutical compounds must be prepared in their optimal crystal forms to maximize their effectiveness, and careful design is needed to make photonic crystals, the periodic arrays of micrometre-sized colloidal particles that should eventually find applications in optical devices.

One of the problems facing the crystal-grower is that crystals often don't form from solution, even when that solution is highly concentrated with the compound to be crystallized. The nineteenth-century physicist J. Willard Gibbs first explained this phenomenon by pointing out that, under conditions where large crystals are stable, the microscopic crystallites - the precursors of bulk crystal formation - are unstable and should disintegrate. But every crystal must start off small, so this implies that large crystals would never form. Brownian motion comes to the rescue: because of the incessant
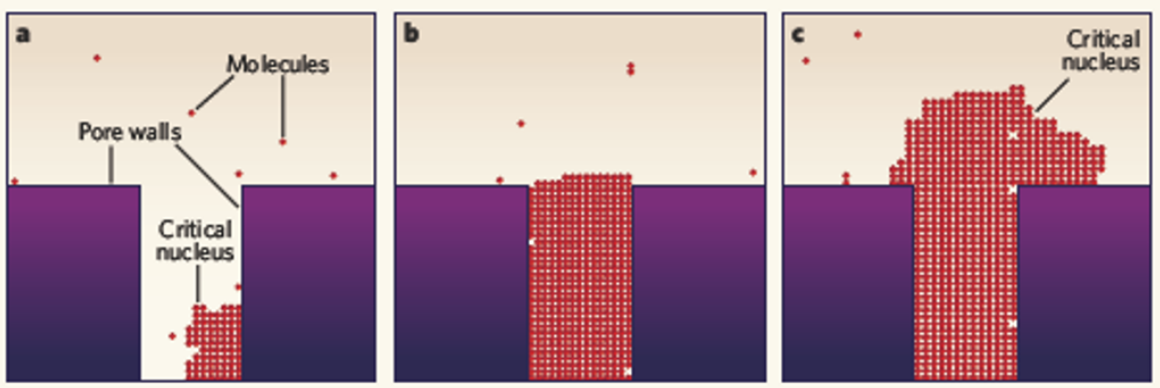

Figure 1 Crystal nucleation in a pore. Page and Sear $^{2}$ propose a model to explain why porous materials induce protein crystallization. a, The pore walls weakly attract the molecules to be crystallized. Crystals start to grow in the corners of the pore when a critical nucleus of molecules forms. b, The crystal grows until the pore is filled. c, Another critical nucleus of molecules forms on the outside of the pore, which allows the 'breakout' of a bulk crystal from the pore. The rates of nucleus formation and breakout vary independently according to the width of the pore. again, this would not favour crystallization.

Now, however, Page and Sear ${ }^{2}$ suggest an explanation for the unexpected crystallization of proteins on a porous surface. They argue that, among the myriad pores at the seed's surface, there will always be some that have exactly the right shape to accommodate a small crystallite. For instance, for a cubic crystal, such a pore would have a rectangular prism shape.

The authors then address the question of what the optimum pore size is for crystal nucleation. The answer is not simple. They consider a highly simplified model in which the width of the pore is easily varied, and find that crystal nudeation proceeds in two stages: first, crystallization starts at the corners of the pore; then, once a crystal has grown to fill the pore, it breaks out to form a bulk crystal (Fig. 1). The rate at which crystals form inside the pore increases as the pore becomes narrower. But the rate at which these crystals go on to form a bulk crystal increases as the pore diameter widens. So, for narrow pores, the breakout time is rate limiting, but for wide pores, nucleation is the slowest step.

The crystallization rate is a a maximum for pore diameters somewhere between the wide and narrow extremes, and it is these optimally sized pores that dominate crystal nucleation on a microporous substrate. The great diversity of pore sizes and shapes in a microporous material increases the likelihood of an optimal pore existing on the surface of that material. In this sense, a microporous material acts as a huge, random library' of crystallization agents.

Understanding the microscopic mechanism behind the success of microporous nucleation agents should help us to design seeds with increased crystallizing power. This can be of considerable practical importance, as the production of high-quality protein crystals is one of the main bottlenecks in the X-ray determination of the structure of water-soluble proteins. Unfortunately, it seems unlikely that microporous seeds will help in crystallizing membrane proteins, which constitute about a third of all proteins.

Microporous materials should be particularly useful as seed substrates for protein crystallization where the aim is to make only a few crystals. Although the vast majority of pores are ineffective, this doesn't matter as long as a few work well. By analogy, we should expect that a great multitude of random substrates - or, more generally, highly diverse environments - are necessary to bring about processes where just one successful event is enough. The evolution oflife may be a case in point. Daan Frenkel is at the FOM Institute for Atomic and Molecular Physics, Kruislaan 407, 1098SJ Amsterdam, The Netherlands. e-mait: frenkel@amolf.nl

1. Chayen, N.E.et al. L.Mol. Biol.312, 591-595(2001).

2. Page, A. I\& Sear, R.P. Phys. Rex Lett. 97,065701 (2006).

3 van Blaaderen, A. et al. Nature 385, 321-324 (1997).

4. Cacciuto, A, Auer, S.\& Frenkel, D. Nature 428, 404-405 (2004). 